\title{
Investigation on the Relationship Between Definition of Suceess, Positiveness of Reaction to Peer Pressure, and Self-perceived Confidence Level
}

\author{
Xingzhi Che ${ }^{1,+}$, Xiwen Guan ${ }^{2,+}$, Xinyi Yin ${ }^{3 *,+}$, Yichun Zhao ${ }^{4,+}$
}

\author{
1Guangzhou Foreign Language School, Guangzhou 511455, China \\ 2Ulink College Guangzhou, Guangzhou 511458, China \\ 3Abington Friends School, Jenkintown, PA 19046, United States \\ 4YK PAO School, Shanghai 201620, China \\ *Corresponding author Email: 22xinyiy@abingtonfriends.net \\ + They are all first authors.
}

\begin{abstract}
This research mainly investigates the correlation between people's definition of success, the positiveness of reaction to peer pressure, and self-perceived confidence level in achieving one's own life goals in the field of social psychology. The influences of culture--China and Japan, gender, and education level on the three factors above were also examined. Questionnaires were utilized to collect data and to draw conclusions about the interplay between the variables. T-tests, ANOVA, and tests for correlations were conducted on SPSS. The results demonstrate that there is a significant correlation between one's definition of success and positiveness of reaction to peer pressure, a significant correlation between positiveness of reaction to peer pressure and one's confidence level, and a significant correlation between one's definition of success and one's self-perceived confidence level. This research report first provides an overview of the literature relevant to the topic, then the research design, methods, and procedures will be explained, and then the analysis of data, the research results, and discussion of the results will be provided; finally, future investigations and policy implications will be suggested.
\end{abstract}

Keywords: definition of success, peer pressure, motivation level, confidence level, Japan and China

\section{INTRODUCTION}

\subsection{Investigation on the Relationship Between Definition of Success, Positiveness of Reaction to Peer Pressure, and Self-perceived Confidence Level}

Culture, defined by diverse components such as religion, belief, knowledge, social habits languages and customs etc. $[1,2]$, is "the collective programming of the mind that distinguishes the members of one group or category of people from others" [3] Unsurprisingly, culture is not firmly bounded, but varies within different historical contexts and societal backgrounds. Just as a study from the University of Rochester has shown, people living in different cultural surroundings may generate multifarious standards of self-fulfillment and values cherishing, which in short, the definitions of success. For that reason, the different definitions of success people hold may more or less have a mutual effect on their confidence level, that is, whether they are able to strive for and achieve the final ambition. In order to find out the correlation between the definitions of success and selfperceived confidence level, a model was created to help develop the research question and the reaction to peer pressure was also involved as a medium of the relationship. To narrow down the concepts of the definitions of success, the ESS scale [4-6] was utilized. In this scale, ten basic human values (self-direction, stimulation, hedonism, achievement, power, security, tradition, conformity, benevolence, and universalism) were derived according to the Value theory [4,5,7]. And according to the Aspiration Index [8,9] which is utilized to measure and classify people's life goals, the notion of "extrinsic aspiration" and "intrinsic aspiration" was reinterpreted and used in the research to categorize people's definition of success (intrinsic life goals: self- 
direction, stimulation, hedonism, security, tradition, conformity, benevolence \& universalism; extrinsic life goals: power \& achievement). As for the positiveness of reaction to peer pressure, the concepts were divided into two parts: motivated to take actions positively and motivated to take actions negatively. Peer pressure, defined as the insistence and encouragement of the same age group individuals to make an individual do something [10], may give students a sense of motivation [11] and eventually influence choices they pursue [12].Despite a lot of research in the past have shown that "values are a motivational construct"[6] and strong self-efficacy can improve an individual's self-control [13], which leads to stronger willpower in achieving success and confidence enhances motivation [14], there still lacks in-depth research to bridge the gaps among the overall definition of success, reaction to peer pressure and self-perceived confidence level that measures how confident people are in achieving their goals. In this case, one of the paper's major purposes is to find out the correlation between the three factors. In addition, as the participants collected are from Japan and China, varying in gender and education levels, some examinations will be done to explore how culture, gender, and education level affected the correlations or the factors.

\subsection{Aim}

The aim of the research is to test and understand the relationships between the definition of success, the positiveness of reaction to peer pressure, and perceived confidence level amongst Chinese and Japanese young people, which is essentially the interaction between attitudes and behaviors. Additionally, the research explored how culture factors, education level, gender may have a possible influence on the three factors: Definition of success, the positiveness of reaction to peer pressure, and perceived confidence level.

\subsection{Rationale}

Once the relationship between the definition of success, the positiveness of reaction to peer pressure, and perceived confidence level are established, and the impact of gender, education level on the three factors above is clear, the method to enhance one's confidence level or improved reaction to peer pressure can be developed. Hence, this research is significant in the way that it may be applied to design the curriculum in school.

\section{RESEARCH}

\subsection{Research Design}

Questionnaires including four sections were sent to participants in Japan and China through social media to collect data. All variables are measured in terms of the Likert Scale and open-ended questions.

\subsection{Hypothesis}

Hypothesis 1: Culture only affects people's definition of success, but not confidence level or positiveness of motivation level when facing peer pressure.

Hypothesis 2: The more one values extrinsic life goals, the more one is motivated by peer pressure positively; there's no correlation between the extent to which one values intrinsic life goals and how positive one is motivated by peer pressure.

Hypothesis 3: The more one is motivated by peer pressure to do positive things, the more confidence one has in general.

Hypothesis 4: The more one values a particular category of life goals, the more confident one is in achieving these life goals.

Hypothesis 5: College students value extrinsic life goals and intrinsic life goals more, and own higher selfperceived confidence levels. (undergraduate students(A); teenagers' cl)

\subsection{Method}

An online questionnaire was used to collect raw data. It was translated into a Chinese version and a Japanese version. The questionnaire was divided into four sections: background information, the definition of success, the positiveness of reaction to peer pressure, and confidence level in achieving success. The first section includes multiple-choice and open-ended questions that ask about participants' gender, current education level, age, and the culture they identify with. Different from the Japanese questionnaire, the Chinese questionnaire included another question about the education system that participants' are currently in (Gaokao or International curriculum) in this section. Within the second section, definitions of success were measured using the ESS scale developed by Schwartz (year). The ESS scale was originally utilized to identify basic values people in all cultures hold. In this research, two aspects of the original scale were revised. One, the basic human values were further divided into two categories--extrinsic and intrinsic--according to the Aspiration Index (Kasser and Ryan, year). Two, the Likert scale's ratings were reduced from 1-6 to 1-4 (1=strongly disagree, 4=strongly agree). In the third section, the positiveness of reaction to peer pressure was measured by the Peer Pressure Inventory (PPI) (name, year). The original form of the Likert Scale rating from 1 to 6 was kept ( $1=$ strongly agree with the statement on the left, $6=$ strongly agree with the statement on the right). The original statements describe possible behaviors under peer pressure but the statements were revised in this research to avoid misinterpretations of ambiguous statements across China and Japan. The fourth section measured participants' confidence level in achieving various life goals--wealth, fame, personal 
growth, affiliation, community involvement, and health. The life goals and the categorization (extrinsic or intrinsic) were defined by the Aspiration Index. The Likert Scale's ratings in this section range from very likely (=4) to very unlikely $(=1)$.

\subsection{Sample}

Convenience sampling was utilized in the research. The intended participants included students from the Shanghai Zizhu International School campus and available contacts on researchers' social media. The sample consisted of 154 participants between the ages of 14 and 24 years $(M=18, S D=2.2)$ from China and Japan. Among the sample group, $56.5 \%$ were female and $43.5 \%$ were male; $61.0 \%$ were currently at high school, $34.4 \%$ were currently at College or University, and $4.5 \%$ were at work. Chinese group included 127(82.5\%) participants and the Japanese group included 27(17.5\%) participants. Among the Chinese group, $37.8 \%$ in the Gaokao curriculum and $62.2 \%$ in the International curriculum.

\subsection{Procedure}

The QR code and link of the questionnaire were sent directly to intended participants using social media (Wechat, QQ, Instagram, Twitter, Facebook). The questionnaire was available for five days and the responses were collected during this period of time. The raw data was automatically generated by Microsoft Forms, and further processing of the data was conducted on Excel, Numbers, and SPSS.

\section{DATA ANALYSIS}

\subsection{Background Analysis}

In the background information section, there are three options for gender--male, female, and others, four options for current education level--high school, college/university, work, and others, one open-ended question about age, four options for the culture respondents identify with--China, Japan, the UK, and the US, and two options for whether one is in the international curriculum in the Chinese questionnaire-yes and no. The answers that choose the option of "others" in any of these sections were later deleted because the sample size for "others" was limited and not representative enough. The answers that exceeded the age limit were also deleted.

\subsection{ESS Scale Analysis}

To examine the extent to which one values a particular category of success, the responses to the ESS Scale were first categorized according to the coding scheme developed by Schwartz which groups the statements according to corresponding basic values. Using the scale of 1 (strongly disagree) to 4 (strongly agree), the total score of each of the basic values was calculated. This total score was divided by the number of corresponding questions so that an average score for each basic value was obtained. The basic values are the subdivisions of the two broad categories--extrinsic life goals and intrinsic life goals. Thus, to calculate the score that can indicate the extent to which one values extrinsic or intrinsic life goals, the scores of subdivisions that belong to the same category were added up and the average was taken. Finally, each participant was given a score that reveals the extent to which he or she values extrinsic and intrinsic life goals respectively, denoted by "ESS ex" and "ESS in" respectively.

To understand whether one is dominated by extrinsic or intrinsic life goals, a final score was calculated by using the extrinsic score to minus the intrinsic score. Thus, if the final score was negative, one values intrinsic goals more than extrinsic goals, and vice versa. The larger the absolute value of the final score is, the greater extent one is dominated by the particular category of life goals. This final score was denoted by "in or ex".

\subsection{PPI Analysis}

The revised version of PPI consists of seven questions in total. For the first six questions, the positive statement was placed on the left side and the negative statement was placed on the right side. Thus, formula 7 minus the original score was used to calculate the final score for each question. The last question acted as a filter question, with the positive statement on the right side and the negative statement on the left side, so the original score of this question equals the final score for this question. The final score of the seven questions was then summed together to obtain the total score that indicates the positiveness of reaction to peer pressure. The higher the score is in this section, the more he or she is motivated to take action positively.

\subsection{Analysis}

A score from 1 (very unlikely) to 4(very likely) was given for each question which asks about one's confidence level in achieving the goal of wealth, fame, personal growth, affiliation, community involvement, and health. The scores for wealth and fame were added up and averaged out to calculate the score for one's confidence level in achieving extrinsic life goals; that for personal growth, affiliation, community involvement, and health were added up and averaged out to calculate the score for one's confidence level in achieving intrinsic life goals. The scores for all of the six questions were added up and averaged out to calculate the confidence level in general. 


\section{RESULTS}

\begin{tabular}{|c|c|c|c|c|}
\hline \multicolumn{5}{|c|}{ Table 1 Independent Samples Test on Culture differences } \\
\hline & & \multirow{2}{*}{ sig. } & \multicolumn{2}{|c|}{ significance } \\
\hline & & & one-sided p & two-sided $p$ \\
\hline \multirow{2}{*}{ ESS extrinsic } & Equal variances assumed & $<.001$ & $<.001$ & $<.001$ \\
\hline & Equal variances not assumed & & $<.001$ & $<.001$ \\
\hline \multirow{2}{*}{ ESS intrinsic } & Equal variances assumed & .002 & $<.001$ & $<.001$ \\
\hline & Equal variances not assumed & & $<.001$ & $<.001$ \\
\hline \multirow{2}{*}{ motiuvation level } & equal variances assumed & .820 & $<.001$ & $<.001$ \\
\hline & Equal variances not assumed & & $<.001$ & $<.001$ \\
\hline \multirow{2}{*}{ in or ex } & Equal variances assumed & $<.001$ & $<.001$ & $<.001$ \\
\hline & Equal variances not assumed & & .004 & .009 \\
\hline \multirow{2}{*}{ cl extrinsic } & Equal variances assumed & .003 & $<.001$ & $<.001$ \\
\hline & Equal variances not assumed & & $<.001$ & $<.001$ \\
\hline \multirow{2}{*}{ Cl intrinsic } & Equal variances assumed & $<.001$ & $<.001$ & $<.001$ \\
\hline & Equal variances not assumed & & .007 & .014 \\
\hline \multirow{2}{*}{ total } & Equal variances assumed & .001 & $<.001$ & $<.001$ \\
\hline & Equal variances not assumed & & $<.001$ & $<.001$ \\
\hline
\end{tabular}

According to table 1, the statistic difference of T-test is less than 0.001 . The data indicated that there are differences between the extent to which participants value extrinsic and intrinsic life goals across cultures, evidenced by $\mathrm{p}<0.001$.

In the T-test of culture and positiveness of reaction to peer pressure, the statistic difference is less than 0.001 , indicating that there are differences between the positiveness of participants' reactions to peer pressure across cultures.

In the T-test of culture and perceived confidence level, the statistic difference is less than 0.001 , indicating that there are differences between participants' selfperceived confidence level of achieving extrinsic life goals. However, there is not enough evidence to support there are differences between participants' self-perceived confidence level of achieving intrinsic life goals and life goals across cultures in general, evidenced by $\mathrm{p}>0.005$.

\subsection{Gender differences}

Table 2 Independent Samples Test on Gender differences

\begin{tabular}{|c|c|c|c|c|}
\hline & & \multirow{2}{*}{ sig. } & siginificance & \multirow[b]{2}{*}{ two-sided p } \\
\hline & & & one-sided p & \\
\hline \multirow{2}{*}{ ESS extrinsic } & Equal variances assumed & $<.001$ & $<.001$ & $<.001$ \\
\hline & Equal variances not assumed & & $<.001$ & $<.001$ \\
\hline \multirow{2}{*}{ ESS intrinsic } & Equal variances assumed & $<.001$ & $<.001$ & $<.001$ \\
\hline & Equal variances not assumed & & $<.001$ & $<.001$ \\
\hline
\end{tabular}

According to table 2, the T-test of Gender and definition of success, the statistic difference is less than 0.001, indicating that different gender leads to a difference in the extent to which participants value extrinsic life goals and intrinsic life goals.

In the T-test gender and positiveness of reaction to peer pressure, the statistic difference greater than 0.001 , indicating that there is not enough evidence to support that different gender leads to a difference in positiveness of reactions to peer pressure.

In the T-test of gender and perceived confidence level, the statistic difference is less than 0.001 , indicating that different gender leads to a difference in participants self-perceived confidence level of achieving extrinsic life goals. 


\subsection{Education level differences}

\begin{tabular}{|c|c|c|c|c|}
\hline \multicolumn{5}{|c|}{ Table 3 Post Hoc Tests on Education Levels } \\
\hline \multicolumn{5}{|c|}{ Multiple Comparions } \\
\hline Dependent Variable & (I) Education Level & (J) Education Level & Mean Difference $(I-J)$ & Sig. \\
\hline \multirow{6}{*}{ ESS extrinsic } & \multirow{2}{*}{1} & 2 & $-1.23379 *$ & $<.001$ \\
\hline & & 3 & -.50266 & .252 \\
\hline & \multirow{2}{*}{2} & 1 & 1.23379 & $<.001$ \\
\hline & & 3 & .73113 & .106 \\
\hline & \multirow{2}{*}{3} & 1 & .50266 & .252 \\
\hline & & 2 & -.73113 & .106 \\
\hline \multirow{6}{*}{ ESS intrinsic } & \multirow{2}{*}{1} & 2 & $-1.460257^{\star}$ & $<.001$ \\
\hline & & 3 & -.50608 & .266 \\
\hline & \multirow{2}{*}{2} & 1 & $1.4602569^{\star}$ & $<.001$ \\
\hline & & 3 & .9541779 & .042 \\
\hline & \multirow{2}{*}{3} & 1 & .5060790 & .266 \\
\hline & & 2 & -.9541779 & .042 \\
\hline \multirow{6}{*}{ motivation level } & \multirow{2}{*}{1} & 2 & $3.764^{\star}$ & $<.001$ \\
\hline & & 3 & 1.357 & .481 \\
\hline & \multirow{2}{*}{2} & 1 & $-3.764^{*}$ & $<.001$ \\
\hline & & 3 & -2.407 & .225 \\
\hline & \multirow{2}{*}{3} & 1 & -1.357 & .481 \\
\hline & & 2 & 2.407 & .225 \\
\hline \multirow{6}{*}{ cl extrinsic } & \multirow{2}{*}{1} & 2 & $.2642^{\star}$ & .016 \\
\hline & & 3 & .2857 & .252 \\
\hline & \multirow{2}{*}{2} & 1 & $-0.2642^{*}$ & .016 \\
\hline & & 3 & .0216 & .933 \\
\hline & \multirow{2}{*}{3} & 1 & -.2857 & .252 \\
\hline & & 2 & -.0216 & .933 \\
\hline \multirow{6}{*}{ total } & \multirow{2}{*}{1} & 2 & $.17536462^{\star}$ & .031 \\
\hline & & 3 & .235562314 & .202 \\
\hline & \multirow{2}{*}{2} & 1 & $-0.1753646^{*}$ & .031 \\
\hline & & 3 & .060197689 & .750 \\
\hline & \multirow{2}{*}{3} & 1 & -.235562310 & .202 \\
\hline & & 2 & -.060297690 & .750 \\
\hline
\end{tabular}

According to table 3, education level and definition of success Post Hoc Test, different education level leads to a difference in the extent to which participants value extrinsic life goals and intrinsic life goals, is evidenced by $\mathrm{p}<0.001$. College students value extrinsic life goals and intrinsic life goals more than high school students, evidenced by the mean difference of 1.23379.

Educational level and positiveness of reaction to peer pressure Post Hoc Test showed that different education level leads to a difference in the motivation level of participants under peer pressure, evidenced by $\mathrm{p}<0.001$. High school students' reaction to peer pressure is more positive than college students, evidence by the mean difference of 3.764 .

Education level and self-perceived confidence level Post Hoc Test showed that, different education level leads to a difference in participant's self-perceived confidence level of achieving extrinsic life goals and life goals in 
general, evidenced by $\mathrm{p}<0.001$. High school students perceive a higher confidence level of achieving extrinsic life goals and life goals in general compared to college students, evidenced by the mean difference of 0.2642 and 0.17536 .

\subsection{Definition of success \& Confidence level correlation}

\begin{tabular}{|c|c|c|c|c|}
\hline \multicolumn{5}{|c|}{ Table 4 Definition of success \& Confidence level in China Correlation } \\
\hline \multicolumn{5}{|c|}{ Correlations } \\
\hline & & ESS extrinsic & ESS intrinsic & motivation level \\
\hline \multirow{2}{*}{ ESS extrinsic } & Pearson Correlation & 1 & $.254^{\star *}$ & $.343^{* *}$ \\
\hline & Sig. (2-tailed) & & .004 & $<.001$ \\
\hline \multirow{2}{*}{ ESS intrinsic } & Pearson Correlation & $.254^{\star \star}$ & 1 & $.200^{*}$ \\
\hline & Sig. (2-tailed) & .004 & & .024 \\
\hline \multirow{2}{*}{ motivation level } & Pearson Correlation & $.343^{\star *}$ & $.200 *$ & 1 \\
\hline & Sig. (2-tailed) & $<.001$ & .024 & \\
\hline
\end{tabular}

\begin{tabular}{|c|c|c|c|c|}
\hline \multicolumn{5}{|c|}{ Table 5 Definition of success \& Confidence level in Japan Correlation } \\
\hline \multicolumn{5}{|c|}{ Correlations } \\
\hline & & ESS extrinsic & ESS intrinsic & motivation level \\
\hline \multirow{2}{*}{ ESS extrinsic } & Pearson Correlation & 1 & $.390 *$ & .083 \\
\hline & Sig. (2-tailed) & & .044 & 679 \\
\hline \multirow{2}{*}{ ESS intrinsic } & Pearson Correlation & $.390 *$ & 1 & .209 \\
\hline & Sig. (2-tailed) & .044 & & .296 \\
\hline \multirow{2}{*}{ motivation level } & Pearson Correlation & .083 & .209 & 1 \\
\hline & Sig. (2-tailed) & .679 & .296 & \\
\hline
\end{tabular}

According to table 5, the data collected from Chinese participants indicates that the correlation between extrinsic value towards success and their motivation level is significant at 0.01 level, showing that there is a moderate correlation according to the table (0.343); and the correlation between intrinsic value towards success and their motivation level is significant at 0.05 level, which shows a weak correlation between the two factors.
The table 6 , by contrast, indicated that responses collected from Japanese participants show that there is no significant correlation between neither extrinsic nor intrinsic value towards success and their motivation level.

\subsection{Confidence level \& Motivation level correlation}

\begin{tabular}{|c|c|c|c|c|c|}
\hline \multicolumn{6}{|c|}{ Table 6 Confidence level \& Motivation Level in China Correlation } \\
\hline \multicolumn{6}{|c|}{ Correlations } \\
\hline & & motivation level & cl extrinsic & Cl intrinsic & total \\
\hline \multirow{2}{*}{ motivation level } & Pearson Correlation & 1 & $.186^{\star}$ & $.343^{* *}$ & $.339 * *$ \\
\hline & Sig. (2-tailed) & & .036 & $<.001$ & $<.001$ \\
\hline \multirow{2}{*}{ cl extrinsic } & Pearson Correlation & $.186^{\star}$ & 1 & $.355^{\star *}$ & $.728 * \star$ \\
\hline & Sig. (2-tailed) & .036 & & $<.001$ & $<.001$ \\
\hline \multirow{2}{*}{$\mathrm{cl}$ intrinsic } & Pearson Correlation & $.343^{\star \star}$ & $.355^{\star \star}$ & 1 & $.899 * *$ \\
\hline & Sig. (2-tailed) & $<.001$ & $<.001$ & & $<.001$ \\
\hline \multirow{2}{*}{ total } & Pearson Correlation & $.339 * *$ & $.728^{\star \star}$ & $.899 * \star$ & 1 \\
\hline & Sig. (2-tailed) & $<.001$ & $<.001$ & $<.001$ & \\
\hline
\end{tabular}




\begin{tabular}{|c|c|c|c|c|c|}
\hline \multicolumn{6}{|c|}{ Table 7 Confidence level \& Motivation Level in Japan Correlation } \\
\hline \multicolumn{6}{|c|}{ Correlations } \\
\hline & & motivation level & cl extrinsic & cl intrinsic & total \\
\hline \multirow{2}{*}{ motivation level } & Pearson Correlation & 1 & .063 & .117 & .109 \\
\hline & Sig. (2-tailed) & & .754 & .560 & .590 \\
\hline \multirow{2}{*}{ cl extrinsic } & Pearson Correlation & .063 & 1 & $.610^{\star \star}$ & $.821^{\star \star}$ \\
\hline & Sig. (2-tailed) & .754 & & $<.001$ & $<.001$ \\
\hline \multirow{2}{*}{ cl intrinsic } & Pearson Correlation & .117 & $.610^{\star \star}$ & 1 & $.953^{* *}$ \\
\hline & Sig. (2-tailed) & .560 & $<.001$ & & $<.001$ \\
\hline \multirow{2}{*}{ total } & Pearson Correlation & .109 & $.821^{\star *}$ & $.953^{* *}$ & 1 \\
\hline & Sig. (2-tailed) & .590 & $<.001$ & $<.001$ & \\
\hline
\end{tabular}

The table 6 demonstrates the correlation between positiveness of reaction to peer pressure and one's confidence level in terms of achieving extrinsic, intrinsic life goals respectively and in terms of achieving life goals in general in China. As indicated by statistics, the correlation between motivation level when facing peer pressure and one's confidence level in achieving extrinsic success is significant at the 0.05 level. There is a weak correlation between people's positiveness of reaction to peer pressure and confidence level in achieving extrinsic life goals, with the Pearson's coefficient being 0.186 . For the correlation between motivation level and confidence level in achieving intrinsic life goals, the correlation is moderate, indicated by the r-value of 0.343 , and the correlation is significant at the 0.01 level. There is a moderate correlation between motivation level and confidence level in general shown by the r-value of 0.339 , and this correlation is significant at the 0.01 level as well.

The same measures were taken for the data from Japan, showing in table 7. However, no significant correlation was found between motivation level and confidence level in achieving extrinsic, intrinsic life goals and life goals in general.

\subsection{Correlation between the definition of success and the confidence level}

\begin{tabular}{|c|c|c|c|c|c|c|}
\hline \multicolumn{7}{|c|}{ Table 8 Correlation between the definitiion of success and the confidence level in China } \\
\hline \multicolumn{7}{|c|}{ Correlations } \\
\hline & & ESS extrinsic & ESS intrinsic & cl extrinsic & cl intrinsic & total \\
\hline \multirow{2}{*}{ ESS extrinsic } & Pearson Correlation & 1 & $.254^{\star *}$ & $.262^{\star \star}$ & 0.073 & $.176^{\star}$ \\
\hline & Sig. (2-tailed) & & .004 & .003 & .416 & .048 \\
\hline \multirow{2}{*}{ ESS intrinsic } & Pearson Correlation & $.254^{\star \star}$ & 1 & .094 & $.297^{* *}$ & $.262^{* *}$ \\
\hline & Sig. (2-tailed) & .004 & & .293 & $<.001$ & .003 \\
\hline \multirow{2}{*}{ cl extrinsic } & Pearson Correlation & $.262^{\star \star}$ & .094 & 1 & $.355^{\star \star}$ & $.728^{* *}$ \\
\hline & Sig. (2-tailed) & .003 & .293 & & $<.001$ & $<.001$ \\
\hline \multirow{2}{*}{$\mathrm{cl}$ intrinsic } & Pearson Correlation & .073 & $.297^{\star \star *}$ & $.355^{\star \star}$ & 1 & $.899 * *$ \\
\hline & Sig. (2-tailed) & .416 & $<.001$ & $<.001$ & & $<.001$ \\
\hline \multirow{2}{*}{ total } & Pearson Correlation & $.176^{\star}$ & $.262^{* *}$ & $.728^{\star \star}$ & $.899 * \star$ & 1 \\
\hline & Sig. (2-tailed) & .048 & .003 & $<.001$ & $<.001$ & \\
\hline
\end{tabular}

\begin{tabular}{|c|c|c|c|c|c|c|}
\hline \multicolumn{7}{|c|}{ Table 9 Correlation between the definitiion of success and the confidence level in Japan } \\
\hline \multicolumn{7}{|c|}{ Correlations } \\
\hline & & ESS extrinsic & ESS intrinsic & cl extrinsic & cl intrinsic & total \\
\hline \multirow{2}{*}{ ESS extrinsic } & Pearson Correlation & 1 & $.390^{\star}$ & .203 & .003 & .079 \\
\hline & Sig. (2-tailed) & & .044 & .310 & ,990 & .695 \\
\hline
\end{tabular}




\begin{tabular}{|c|l|r|r|r|r|r|}
\hline \multirow{2}{*}{ ESS intrinsic } & Pearson Correlation & $.390^{*}$ & 1 & .077 & .246 & .207 \\
\cline { 2 - 6 } & Sig. (2-tailed) & .044 & & .703 & .215 & .300 \\
\hline \multirow{3}{*}{ cl extrinsic } & Pearson Correlation & .203 & .077 & 1 & $.610^{\star *}$ & $.821^{\star \star}$ \\
\cline { 2 - 6 } & Sig. (2-tailed) & .310 & .703 & & $<.001$ & $<.001$ \\
\hline \multirow{2}{*}{ cl intrinsic } & Pearson Correlation & .003 & .246 & $.610^{\star *}$ & 1 & $.953^{\star \star}$ \\
\cline { 2 - 7 } & Sig. (2-tailed) & .990 & .215 & $<.001$ & $<.001$ \\
\hline \multirow{2}{*}{ total } & Pearson Correlation & .079 & .207 & $.821^{\star \star}$ & $.953^{\star *}$ & 1 \\
\cline { 2 - 7 } & Sig. (2-tailed) & .695 & .300 & $<.001$ & $<.001$ & \\
\hline
\end{tabular}

To understand the relationship between perception of success \& confidence level in China, four correlations in table 8 should be looked at. Firstly, the correlation between the extent to which young people value extrinsic life goals and their confidence level in achieving extrinsic life goals is significant at the 0.01 level. It is a weak correlation at 0.176. Secondly, the correlation between the extent to which young people value intrinsic life goals and their confidence level in achieving intrinsic life goals is significant at the 0.01 level. It is a weak correlation at 0.297 .

Thirdly, the correlation between the extent to which young people value extrinsic life goals and their confidence level in achieving life goals, in general, is significant at the 0.05 level. It is a weak correlation at 0.176 . Lastly, the correlation between the extent to which young people value intrinsic life goals and their confidence level in achieving life goals, in general, is significant at the 0.01 level. It is a weak correlation at 0.262 .

Overall, when participants value a particular aspect, they are more confident at this aspect.

\subsection{Culture and Gender trends}

\begin{tabular}{|c|c|c|c|c|c|c|}
\hline & power & achievement & self-direction & stimulation & hedonism & security \\
\hline Japanese Male ESS & 2.6 & 3.3 & 3.5 & 3.5 & 3.8 & 3.6 \\
\hline Japanese Female ESS & 2.7 & 3.1 & 3.2 & 2.7 & 3.6 & 3.7 \\
\hline Chinese Male ESS & 2.8 & 3.1 & 3.2 & 2.8 & 3.1 & 3.3 \\
\hline Chinese Female ESS & 2.9 & 3.0 & 3.2 & 2.8 & 3.3 & 3.4 \\
\hline
\end{tabular}

\begin{tabular}{|c|c|c|c|c|c|c|c|c|c|c|c|}
\hline \multicolumn{12}{|c|}{ Table 11Culture and Gender trends continued } \\
\hline tradition & \multicolumn{2}{|c|}{ comformity } & benevolence & \multicolumn{2}{|c|}{ universalism } & \multicolumn{2}{|c|}{ ESS extrinsic } & \multicolumn{2}{|c|}{ ESS intrinsic } & \multicolumn{2}{|c|}{ in or ex } \\
\hline 2.3 & & 2.8 & 3.4 & & 3.8 & & 2.9 & & 3.3 & & -0.4 \\
\hline 2.5 & & 3.0 & 3.5 & & 3.5 & & 2.9 & & 3.2 & & -0.3 \\
\hline 2.2 & & 2.8 & 3.3 & & 3.3 & & 2.9 & & 3.0 & & -0.1 \\
\hline \multirow[t]{2}{*}{2.2} & & 2.9 & 3.3 & & 3.3 & & 3.0 & & 3.0 & & -0.1 \\
\hline & & wealth & fame & $\begin{array}{l}\text { personal } \\
\text { growth }\end{array}$ & afflia & ation & community & health & $\begin{array}{l}\mathrm{Cl} \\
\text { extrir }\end{array}$ & & cl intrinsic \\
\hline \multicolumn{2}{|c|}{ Japanese Male CL } & 3.0 & 3.0 & 4.0 & & 3.3 & 4.0 & 3.7 & & 3.0 & 3.8 \\
\hline \multicolumn{2}{|c|}{$\begin{array}{l}\text { Japanese Female } \\
\mathrm{CL}\end{array}$} & 2.4 & 2.0 & 3.0 & & 2.9 & 2.8 & 2.7 & & 2.2 & 2.8 \\
\hline \multicolumn{2}{|c|}{ Chinese Male CL } & 3.2 & 3.0 & 3.6 & & 3.3 & 3.0 & 3.3 & & 3.1 & 3.3 \\
\hline \multicolumn{2}{|c|}{ Chinese Female CL } & 3.1 & 2.8 & 3.5 & & 3.4 & 2.9 & 3.3 & & 3.0 & 3.3 \\
\hline
\end{tabular}

In terms of trends of the definition of success, as showed in table 10, Japanese participants value intrinsic life goals more than Chinese participants by 0.35 point scale.
In terms of confidence level, Japanese female has significant lower confidence level overall compare to other participants. Although Japanese females demonstrate lower confidence level in general compared 
to other groups, their confidence level in affiliation differs less from other groups compared to the difference in other aspects of success in the comparisons with other groups. To conclude, Japanese female is less unconfident in achieving their life goal of affiliation.

\section{DISCUSSION}

\subsection{Culture differences (T-test and trend)}

Firstly, for the reason why the Japanese sample value more intrinsic life goals than Chinese participants"Chinese youths are often also pressured by their parents to choose an education and work that will generate a relatively high salary and this can be explained by the fact that the retirement system in China is weak and that the parents must rely on their children in order to survive economically." Chinese young people preferred material requirements and realistic elements in seeking a university or workplace since a prevalent phenomenon in China-- children give economic support to their parents as the result of rising up and moral values. "Chinese sample comes out highest on material concerns, and equally and relatively low on social and intrinsic factors." Compared to Chinese, Japanese value harmonious relationships with family and friends, especially in a high-conformity society. And Japanese intend to avoid divorces and obtain a harmonious marriage in order to behave "properly".

Secondly, for the reasons why Japanese has a lower self-perceived confidence level. Japanese has a lower self-perceived confidence level of achieving life goals in general compared to Chinese. The reason can be that most of the participants from the Japanese sample are females, and further discussion of the confidence in females is included in the next section.

\subsection{Gender differences(t-test and trend)}

Firstly for the reasons why Japanese females are not confident.The perceived confidence level amongst Chinese males and females were similar. While Japanese data present extremely difference between males and females, which females perceive remarkable low confidence level of achieving extrinsic life goals and intrinsic life goals. "Within the relationship itself, a woman's worth in society is based upon the status of her husband or male partner, leaving much of the power in his hands [15].", Japanese women are endowed with the worth of becoming a good housewife for a successful man instead of exhibiting their abilities in the workplace. Japanese women felt the lack of social support in the workplace due to the stereotype built by society, then their confidence level will decline.

Secondly, the explanation for the T-test results (gender and the extent to which one values a particular aspect of life goals). Although the T-test results have demonstrated a statistically significant difference between the extent to which males and females in general value a particular aspect of life goals, further calculations on the actual average score of males and females in the definition of success part have shown that the difference is negligible, as the two groups obtained similar results on ESS extrinsic and ESS intrinsic scale. Thus, there is still not enough evidence to show that gender can affect one's life goals in general.

\subsection{Education Level (Anova)}

The results match the hypothesis, however, there is a difference in college students' self-perceived confidence level. The data indicated that high school students have a higher perceived confidence level. In terms of high school students, especially for children in Eastern culture, they are protected by parents from dark sides in society and did not encounter many obstacles which would lead to a blow to their self-confidence.

\subsection{Chinese participants}

\section{China (definition of success and confidence level)}

For the reason why college students have a lower confidence level is like bellow. The results match the hypothesis, however, there is a difference in college students' self-perceived confidence level. The data indicated that high school students have a higher perceived confidence level. In terms of high school students, especially for children in Eastern culture, they are protected by parents from dark sides in society and did not encounter many obstacles which would lead to a blow to their self-confidence.

\section{China (Definition of success and confidence level)}

More competition nowadays in all aspects of life, and young people's choices are crucial in deciding their life paths in the future - although they may hold intrinsic life goals in the long run, they need to face the competition in the short term, so they are affected by peer pressure to a certain extent. However, the correlation is less significant for people with intrinsic life goals than those with extrinsic life goals, which fits our hypothesis.

\section{China (confidence level and motivation level)}

The positive actions one takes when being motivated by peer pressure can help with one's personal growth as one is constantly improving himself/herself, which echoes with intrinsic life goals more directly.

\section{China (definition of success and motivation level)}

The results obtained confirmed the hypothesis that the more one values a particular aspect of life goals, the more confidence one has in achieving it. 


\subsection{Japanese Participants}

Since no significant correlation between A, B, and C was found in Japan, all three hypotheses were not supported by our data. Our model did not apply to Japan, mainly because of the non-representative sample. Japanese group only consisted of 27 participants, and $14.8 \%$ of them were female. In addition to this, there can be different interpretations of peer pressure across cultures. While Chinese culture focuses more on competition, Japanese culture focuses more on peer conformity to fit in and maintain harmony within the group.

\subsection{Limitation}

Firstly, the convenience sampling technique led to reduce the chance of the sample being representative. Because participants who responded to our survey imply they at least have an interest in answering the survey. These people may prone to academic success in answering the definition of success questions. But this may differ from the general situation in the young people population. Hence, the result of the study may not accurately reflect reality, and the generalizability of the research is limited. Secondly, because no access to the official translation of the ESS scale and PPI, the translation of questionnaires was done by the researcher. During the translation process, some statements were localized to help with understanding. But there can be divergences in understanding across the culture. Thirdly, since there are more than 70 questionnaires, participants may experience fatigue effect. In fact, some participants commented that he and she were not concentrated at last section of the survey. Additionally, the social desirability effect may influence the answers to PPI as there is a possibility for participants to choose the socially desirable answers instead of their honest responses. Also, in order to access samples outside the campus and culture, online questionnaires were used. However, this means there is no control over the environment of participants answering the survey. This might increase the chance of confounding variables affecting the result. Closed-ended questions may force answer as well. Lastly, because the aim of the study was clearly stated at the begging of the survey, demand characteristics may affect participants' answers.

\subsection{Improvement}

Firstly, to increase the generalizability of the study, a stratified sampling technique should be used to improve the representativeness. Also, the sample should include more participants from Japanese culture to have an equal number of participants in each condition of culture. Hence the result of the study would be more accurate. Secondly, offline questionnaire should be used- the researcher will choose a time and place to supervise participants when participants fill in the questionnaires. By standardizing the procedure, the chance of confounding variables affecting participants' responses will be reduced. Thirdly, ppen-ended questions can be added at the end of the questionnaires to allow participants to express their opinion using their own words instead of choosing limited answers. This will avoid forcing answers.

\subsection{Further Investigations}

\section{Western culture}

The results indicated that culture affects three dimensions among young people in Eastern countries, so further research about Western cultures could be conducted. Since in ESS questionnaire, there is no question about how young people perceive success in terms of image while image is included in aspiration index. In terms of further research, people's perception of success in terms of image could be studied.

\section{Different age group}

Some study shows that there is a great difference in how people perceive success and self-perceived confidence level among various age groups. Further study could be conducted among different age ranges.

\section{Difference in motivation level and reasons across culture}

Although the differences in motivation level and confidence level across China and Japan lack enough evidence to support, we found that the average positive motivation level of China and Japan differ by approximately 1 point which is not negligible considering the scale which is 1 to 6 , and both of the standard deviations is around 0.6 which indicate that most participants agree on the level of positive motivation stimulated by peer pressure ; thus, further investigations can be done in terms of whether there were such differences, as well as the reasons behind such differences.

\subsection{Policy}

The results of the research can be applied to the field of education. If teachers are able to activate students' willingness to achieve the life goal or increase the extent to which they value their own life goals, then students may demonstrate a more positive reaction to peer pressure and more confidence in achieving success. Females, especially Japanese females, need to be encouraged to pursue their life goals as they have less confidence currently. Overall, the research paves a new path for methods in increasing confidence level in achieving success in general. 


\section{CONCLUSION}

The investigation has demonstrated that, in China there is significant correlation between people's definition of success and their confidence level, between people's positiveness of reaction to peer pressure and confidence level, between one's definition of success and confidence level. While in Japan, there is no significant correlation witnessed between any of the two factors.

The results of the research are largely applicable to the field of education. If teachers are able to activate students' willingness to achieve the life goal or increase the extent to which they value their own life goals, then students may demonstrate a more positive reaction to peer pressure and more confidence in achieving success. Females, especially Japanese females, need to be encouraged to pursue their life goals as they have less confidence currently. Overall, the research paves a new path for methods in increasing confidence level in achieving success in general.

Further investigations can be extended to not only Eastern but Western culture, and to different age groups. In addition, it is found that although the differences in motivation level and confidence level across China and Japan lack enough evidence to support, the numerical values differ by approximately 1 point which is not negligible considering the scale which is 1 to 6 , and both of the standard deviations is around 0.6 which indicate that most participants agree on the level of positive motivation stimulated by peer pressure. Thus, further research is required to examine the reasons behind such difference.

\section{REFERENCES}

[1] Flisak D. \& Bjerkhage T. (2015) How culture affects the motivation of employees-A study in differences in motivation between Swedish and Chinese employees http://hdl.handle.net/2077/39667

[2] Tylor. B. Edward. 1871, Researches Into the Development of Mythology, Philosophy, Religion, Art, and Custom(electronic) 1th edition: London: Bradbury, Evans, and CO, Printers, Whitefriars, chapter1, p.1

[3] Hofstede, G. 2011. Dimensionalizing cultures: The Hofstede model in context', Psychology and Culture, vol. 2, no. 1, chapter 8. p.3

[4] Schwartz, S. H. (1992). Universals in the content and structure of values: Theory and empirical tests in 20 countries. In M. Zanna (Ed.), Advances in experimental social psychology (Vol. 25) (pp. 1-65). New York: Academic Press.

[5] Schwartz, S. H. (2005a). Basic human values: Their content and structure across countries. In A. Tamayo
\& J. B. Porto (Eds.), Valores e comportamento nas organizações [Values and behavior in organizations] pp. 21-55. Petrópolis, Brazil: Vozes.

[6] Schwartz, S. H. (2012). An Overview of the Schwartz Leory of Basic Values. Online Readings in Psychology and Culture, 2 (1) https://doi.org/10.9707/2307-0919.1116

[7] Schwartz, S. H. (2006). Basic human values: Theory, measurement, and applications. Revue française de sociologie,

[8] Kasser, T. \& Ryan, R. M. (1993). A dark side of the American dream: correlates of fifinancial success as a central life aspiration. Journal of Personality and Social Psychology, 65(2),410 422.

[9] Kasser, T. \& Ryan, R. M. (1996). Further examining the American dream: differential correlates of intrinsic and extrinsic goals. Personality and Social Psychology Bulletin, 22(3), 280287. doi: $10.1177 / 0146167296223006$

[10] Santor, D., Messervey, D. and Kusumakar, V. (2000). Measuring peer pressure, popularity, and conformity in adolescent boys and girls: predicting school performance, sexual attitudes, and substance abuse. Journal of Youth and Adolescence, 29(2), 165 .

[11] Sukkyung, Y., (2011) Peer influence and adolescents' school engagement, Elsevier Ltd., doi:10.1016., p.1

[12] Owoyele, J. W. and Toyobo, O.M. (2008). Parental will, peer pressure, academic ability and school subjects selection by students in senior secondary schools. The Social Sciences 3 (8): 583-586.

[13] Bandura, A., (1997) Self Efficacy: The Exercise of Control. W.H. Freeman Company, New York

[14] Bénabou R., Tirole J., (2001) Self-confidence and Personal Motivation https://academic.oup.com/qje/articleabstract/117/3/871/1933029

[15] Lebra, T. (2007). Collected papers of Takie Lebra: Identity, gender, and status in Japan. (Vol. 2). Folkestone, UK: Global Oriental Ltd. 